\title{
Studies on the Complexation of Succinic Hydrazide with Copper Chloride Salt
}

\author{
Shereen. E. Salem, Esam A. Gomaa, Mohamed M. El-Defraway, Noha. M. Mohamed
}

\section{ABSTRACT}

The electrochemical behavior of the complexation between copper chloride salt and succinic hydrazide can be explained using cyclic voltammetric measurements. The complex is formed through the interaction with nitrogen and hydroxyl group or carbonyl group of succinic hydrazides. This interaction can be observed by decreasing in the height peak of current and measuring the (anodic/cathodic) shift of the potentials. All the solvation and thermodynamic parameters for the interaction of copper ions with succinic hydrazide as stability constant, Gibbs free energies, enthalpies and entropies of interaction were calculated. Finally, the activity of the formed complex was compared with the succinic hydrazide by comparing their effects on different types of gram-negative bacteria and fungi indicating high activity of the formed complex and its ability to be used in different medical applications.

Keywords: Succinic hydrazide, Complexation, Cyclic voltammetry, Solvation and Kinetic parameters, Stability constant, Antimicrobial activity.

\section{INTRODUCTION}

Electroanalytical techniques have been found a wide application in the characterization of the formed complex in a solution. Cyclic voltammetry is the first experiment performed in an electrochemical study of a compound, biological material or an electrode surface due to its capability for rapidly observing the redox behavior over a wide potential range [1-3].

Organic chemists used this technique to study the electrochemically generated free radicals while inorganic chemists used it to evaluate the effects of ligands on the oxidation and reduction potential of the central metal ion in complexes. This information can be useful for nonanalytical purposes as the studies of the oxidation and reduction process, adsorption process in various media and electron transfer processes at chemically modified electron surfaces [4-7].

In our work, we use the cyclic voltammetry to study the interaction between copper ions and succinic hydrazide in $0.1 \mathrm{M} \mathrm{HCl}$ using solid glassy carbon electrode and scan rate $0.1 \mathrm{~V} / \mathrm{Sec}$. The complex formation was observed by the change in peak heights and shift in the anodic and cathodic couples of peaks of multivalent copper ions.
Published Online: February 11, 2021

ISSN: $2684-4478$

DOI :10.24018/ejchem.2021.2.1.40

\section{S. E. Salem*}

Chemistry Department, Faculty of

Science, Mansoura University, Mansoura, Egypt.

(e-mail: sara_salem669@yahoo.com)

Esam A. Gomaa

Chemistry Department, Faculty of

Science, Mansoura University, Mansoura, Egypt.

(e-mail: eahgomaa65@yahoo.com)

Mohamed M. El-Defraway

Chemistry Department, Faculty of

Science, Mansoura University, Mansoura,

Egypt.

(e-mail: mmeldefrawy@yahoo.com)

Noha. M. Mohamed

Chemistry Department, Faculty of

Science, Mansoura University, Mansoura,

Egypt.

(e-mail: said158456@gmail.com)

*Corresponding Author

\section{EXPERIMENTAL}

\section{A. Materials and Solvents}

Copper chloride salt $\left(\mathrm{CuCl}_{2} .2 \mathrm{H}_{2} \mathrm{O}\right)$ with $\mathrm{Mol}$.Wt equal $170.48 \mathrm{~g} / \mathrm{mol}$, Succinic hydrazide as a ligand and ethanol $(\mathrm{EtOH})$ as analytical reagent grade material were used in this study and all from Merck Company. Hydrochloric acid pure $(\mathrm{HCl})$ from Adwic Co. and bidestilled water was prepared in our laboratory with conductivity of $3 \mu \mathrm{S} \mathrm{cm}^{-1}$.

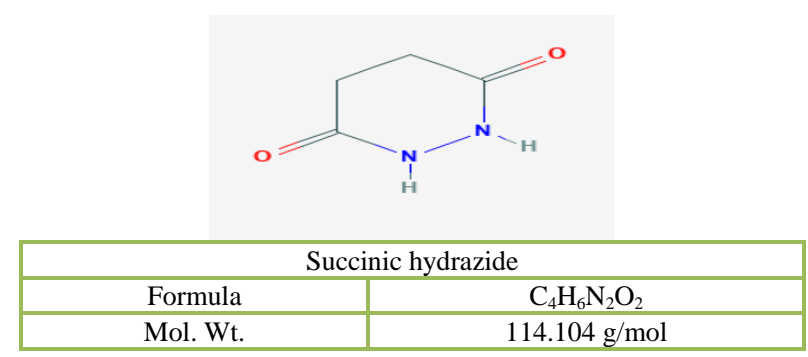

\section{B. Preparation of Copper Complex}

A traditional method was used to prepare the copper complex by refluxing $1 \mathrm{mmol}$ of $\mathrm{CuCl}_{2} \cdot 2 \mathrm{H}_{2} \mathrm{O}$ salt under investigation with $1 \mathrm{mmol}$ of Succinic hydrazide using an ethanolic solution for $2-3 \mathrm{~h}$ close to the boiling point of the solvent. Then, the precipitate was filtered off, washed 
several times with absolute ethanol and dried in vacuum desiccators over anhydrous calcium chloride. Finally, the complex was characterized by using different techniques.

\section{Cyclic Voltammetry}

The cyclic voltametric studies were done by using DY2000 multichannel potentiometer, delivered from USA. It was connected to a cell of three electrodes; silver/silver chloride put in saturated $\mathrm{KCl}$ solution used as reference electrode, solid glassy Carbon electrode (SGC) was used as working electrode and platinum wire as auxiliary electrode. The solid glassy Carbon electrode (SGR) surface was polished to mirror state using (1-0.03) $\mu$ alumina powder. The SGR was washed with absolute alcohol and doubly distilled water till removing any adhering alumina particles. Area of electrode is $\left(7.068 \times 10^{-2}\right) \mathrm{cm}^{2}$. The system was applied from (1 to -1$) \mathrm{V}$ potential window and $(0.1,0.02$, $0.01)$ V.s ${ }^{-1}$ scan rates. Passing purified $\mathrm{N}_{2}$ was done before each experiment to insure inert atmosphere and diffusion experiment. Finally, the data was analyzed using origin software.

\section{Biological Activity (Antibacterial and Antifungal) Activity}

The antimicrobial activities of Succinic hydrazide and its complex were studied on LB agar by the disc diffusion technique against clinical isolates of gram-negative bacteria (Enterobacter, Staphylococcus aureus) and Fungi (Candida albicans).

Sterile filter paper discs $(6 \mathrm{~mm})$ were individually immersed in Dimethylsulfoxide (DMSO) extract of Succinic hydrazide, copper complex and DMSO was taken as control. All the discs were dried, placed on the surface of the test bacterial and fungal and incubated for 18 to $24 \mathrm{~h}$ at $37^{\circ} \mathrm{C}$. The standard antibiotic used is Ceftazidime $(30 \mathrm{mg})$ and finally the size of inhibition zones was measured.

\section{RESULTS AND DISCUSSION}

\section{A. Infrared Analysis (IR)}

Infrared spectra (IR) are an important and popular tool for structural elucidation and compound identification. IR of Succinic hydrazide and its complex with copper chloride salt were obtained by detecting changes in transmittance (or absorption) intensity as a function of frequency (Fig. 1, 2).

From IR spectra of Succinic hydrazide two symm (-NH) group appear at wavenumber (3312 and 3287) $\mathrm{cm}^{-1}$, respectively. Also, two amidic $(\mathrm{C}=\mathrm{O})$ appear at wavenumber (1627 and 1608) $\mathrm{cm}^{-1}$, respectively and $(\mathrm{N}-\mathrm{H})$ bend appear at $1532 \mathrm{~cm}^{-1}$ (Fig. 1).

In IR spectra of copper complex indicates presence of a broad band at $3481 \mathrm{~cm}^{-1}$ due to water molecules, the two symm (-NH) group shifted to lower wavenumber (3234 and $3137) \mathrm{cm}^{-1}$, respectively. One amidic $(\mathrm{C}=\mathrm{O})$ shifted to higher wavenumber $1635 \mathrm{~cm}^{-1}$ while the other amidic $(\mathrm{C}=\mathrm{O})$ shifted to lower wavenumber $1579 \mathrm{~cm}^{-1}$ and the $(\mathrm{N}-\mathrm{H})$ bend appear at $1542 \mathrm{~cm}^{-1}$ (Fig. 2). This indicates the formation of complex between $\mathrm{CuCl}_{2}$ salt and Succinic hydrazide.

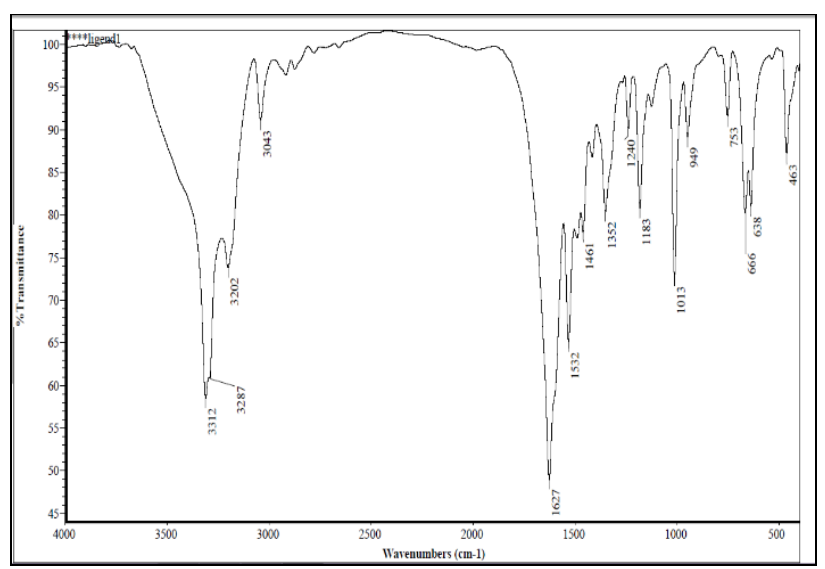

Fig. 1. IR Spectra of Succinic hydrazide.

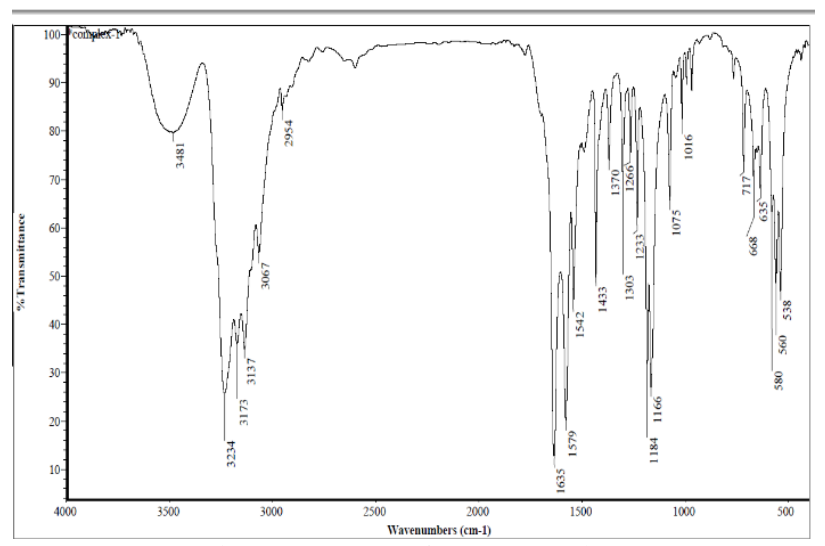

Fig. 2. IR Spectra of copper complex.

\section{B. Cyclic Voltammetry (CV)}

1. Solvation and kinetic parameters in absence of Succinic hydrazide

1.1. Effect of different copper ions concentrations alone

The redox behavior of $\mathrm{Cu}^{2+}$ ions was examined in $30 \mathrm{ml}$ $\mathrm{HCl}(0.1) \mathrm{M}$ as a supporting electrolyte from (1 to -1$) \mathrm{V}$ of potential window and the measured current unit is Ampere, whereas the scan is $0.1 \mathrm{~V} / \mathrm{S}$ (volt per second) at $290.65 \mathrm{~K}$ (Fig. 3). The copper chloride solution is added step wisely from $0.5 \mathrm{ml}\left(1.64 \times 10^{-3}\right) \mathrm{M}$ to reach $1.6 \mathrm{ml}\left(5.06 \times 10^{-3}\right) \mathrm{M}$ (Fig. 4).

The electrochemical redox behavior of $\mathrm{Cu}^{2+}$ ions at the solid glassy carbon electrode was studied at the steady state current, cyclic waves were obtained and explained using the following equations [8-18]:

$\mathrm{i}_{\mathrm{p}}=0.4463 \mathrm{nFAC}(\mathrm{n} \mathrm{F} \mathrm{D} v / \mathrm{R} \mathrm{T})^{1 / 2}$

$\Delta \mathrm{E}_{\mathrm{P}}=\mathrm{E}_{\mathrm{Pa}}-\mathrm{E}_{\mathrm{Pc}}$

$\mathrm{k}_{\mathrm{s}}=2.18 *\left[\mathrm{D}_{\mathrm{C}} \alpha \mathrm{n}_{\mathrm{a}} \mathrm{F} v / \mathrm{RT}\right]^{1 / 2} * \exp \left[\alpha^{2} \mathrm{nF} \Delta \mathrm{E}_{\mathrm{P}} / \mathrm{RT}\right]$

$\alpha \mathrm{n}_{\mathrm{a}}=1.857 \mathrm{RT} /\left(\mathrm{E}_{\mathrm{pc}}-\mathrm{E}_{\mathrm{pc} / 2}\right) \mathrm{F}$

$\Gamma=\mathrm{i}_{\mathrm{p}} 4 \mathrm{RT} / \mathrm{n}^{2} \mathrm{~F}^{2} \mathrm{~A} v$

$\mathrm{Q}=\mathrm{nFA} \Gamma$

where, $i_{p}$ is the current in Ampere, $A$ is the surface area of working electrode in $\mathrm{cm}^{2}, \mathrm{D}$ is the diffusion coefficient in $\mathrm{cm}^{2} / \mathrm{Sec}, \quad v$ is the scan rate in volts/Sec, $\mathrm{C}$ is the 
concentration of the $\mathrm{Cu}^{2+}$ ions, $\Delta \mathrm{E}_{\mathrm{P}}$ is the peak potential difference, $\mathrm{n}$ is the number of electrons in redox reactions, $\mathrm{k}_{\mathrm{s}}$ is the standard heterogeneous electron transfer rate constant in $\mathrm{cm} / \mathrm{sec}, \alpha$ is charge transfer coefficient, $\mathrm{n}_{\mathrm{a}}$ is the numbers of electron transfer in the rate determining step, $\mathrm{E}_{\mathrm{pc} / 2}$ is the half wave potential for cathodic peak, $\Gamma$ is the surface coverage in mol.cm ${ }^{-2}$ and $Q$ is the quantity of charge consumed during the reduction or adsorption of the adsorbed layer.

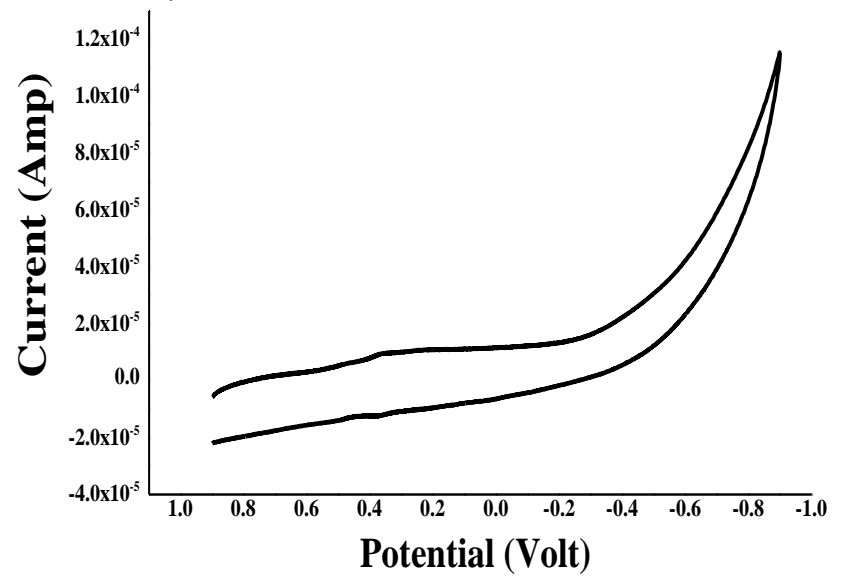

Fig. 3. Cyclic voltammogram of $30 \mathrm{ml} \mathrm{HCl}(0.1) \mathrm{M}$ and scan rate $0.1 \mathrm{~V} / \mathrm{S}$ at $290.65 \mathrm{~K}$.

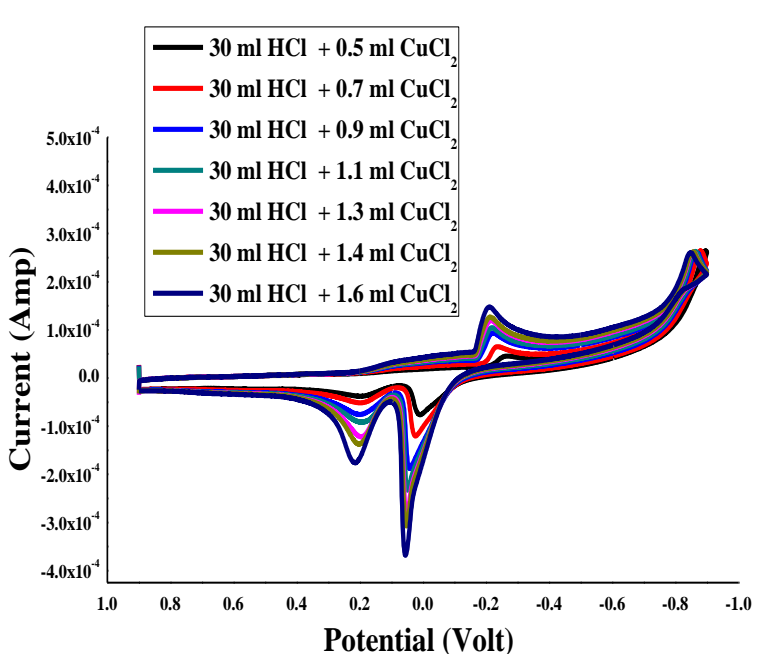

Fig. 4. Cyclic voltammogram of different $\mathrm{Cu}^{2+}$ ions concentrations in $30 \mathrm{ml}$ $\mathrm{HCl}(0.1) \mathrm{M}$ and scan rate $0.1 \mathrm{~V} / \mathrm{S}$ at $290.65 \mathrm{~K}$

The different cyclic voltametric data $\left[\mathrm{Ep}_{\mathrm{a}}\right.$ (anodic peak potential), $\mathrm{Ep}_{\mathrm{c}}$ (cathodic peak potential),Ip (anodic peak current), $\mathrm{Ip}_{\mathrm{c}}$ (cathodic peak current), $\Delta \mathrm{E}_{\mathrm{P}}$ (peak potential difference), $\mathrm{E}_{\mathrm{pc} / 2}$ (half wave potential), $\mathrm{D}_{\mathrm{a}}$ ( anodic diffusion coefficient), $\mathrm{D}_{\mathrm{c}}$ (cathodic diffusion coefficient), $\mathrm{k}_{\mathrm{s}}$ ( electron transfer rate constant), $\Gamma_{\mathrm{a}}$ (anodic surface coverage), $\Gamma_{\mathrm{c}}$ (cathodic surface coverage), $\mathrm{Q}_{\mathrm{a}}$ (anodic quantity of electricity) and $\mathrm{Q}_{\mathrm{c}}$ (cathodic quantity of electricity)] were calculated in Table I (A,B).

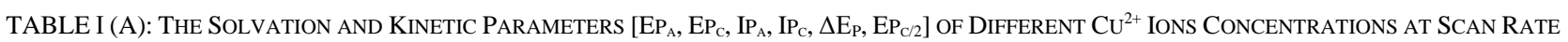

\begin{tabular}{cccccccc}
\hline \multicolumn{7}{c}{$0.1 \mathrm{~V} / \mathrm{S}$ AND $290.65 \mathrm{~K}$} \\
\hline $\mathrm{ml} \mathrm{0f} \mathrm{M}$ & {$[\mathrm{M}] \times 10^{-3}\left(\mathrm{~mol} . \mathrm{L}^{-1}\right)$} & $\mathrm{Ep}_{\mathrm{a}}(\mathrm{V})$ & $\mathrm{Ep}_{\mathrm{c}}(\mathrm{V})$ & $(-) \mathrm{Ip}_{\mathrm{a}} \times 10^{-4}(\mathrm{~A})$ & $\mathrm{Ip}_{\mathrm{c}} \times 10^{-5}(\mathrm{~A})$ & $\Delta \mathrm{E}_{\mathrm{p}}(\mathrm{V})$ & $\mathrm{Ep}_{\mathrm{c} / 2}(\mathrm{~V})$ \\
\hline 0.5 & 1.64 & 0.0044 & 0.26332 & 0.687 & 2.16 & 0.259 & 0.2398 \\
0.7 & 2.28 & 0.022 & 0.235616 & 1.10 & 3.49 & 0.2136 & 0.2166 \\
0.9 & 2.91 & 0.0411 & 0.227596 & 1.75 & 5.34 & 0.1865 & 0.2012 \\
1.1 & 3.54 & 0.0487 & 0.218511 & 2.26 & 6.17 & 0.1698 & 0.197 \\
1.3 & 4.15 & 0.0518 & 0.217647 & 2.78 & 7.36 & 0.1658 & 0.1885 \\
1.4 & 4.46 & 0.0512 & 0.211839 & 3.09 & 7.61 & 0.1606 & 0.1868 \\
1.6 & 5.06 & 0.0553 & 0.208432 & 3.57 & 9.47 & 0.1532 & 0.1841 \\
\hline
\end{tabular}

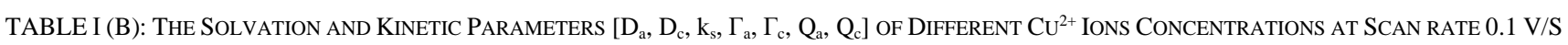

\begin{tabular}{|c|c|c|c|c|c|c|c|c|}
\hline $\mathrm{ml}$ of $\mathrm{M}$ & $\begin{array}{l}{[\mathrm{M}] \times 10^{-3}} \\
\left(\mathrm{~mol} . \mathrm{L}^{-1}\right)\end{array}$ & $\begin{array}{l}\mathrm{D}_{\mathrm{a}} \times 10^{-7} \\
\left(\mathrm{~cm}^{2} \cdot \mathrm{s}^{-1}\right)\end{array}$ & $\begin{array}{l}\mathrm{D}_{\mathrm{c}} \times 10^{-9} \\
\left(\mathrm{~cm}^{2} \cdot \mathrm{s}^{-1}\right)\end{array}$ & $\begin{array}{c}\mathrm{k}_{\mathrm{s}} \mathrm{C} \\
\left(\mathrm{cm} \cdot \mathrm{s}^{-1}\right)\end{array}$ & $\begin{array}{c}\Gamma_{\mathrm{a}} \times 10^{-7} \\
\left(\mathrm{~mol} / \mathrm{cm}^{2}\right)\end{array}$ & $\begin{array}{c}\Gamma_{\mathrm{c}} \times 10^{-7} \\
\left(\mathrm{~mol} / \mathrm{cm}^{2}\right)\end{array}$ & $\begin{array}{c}(-) \mathrm{Q}_{\mathrm{a}} \\
\times 10^{-4}(\mathrm{C}) \\
\end{array}$ & $\begin{array}{c}(+) \mathrm{Q}_{\mathrm{c}} \\
\mathrm{x} 10^{-5}(\mathrm{C}) \\
\end{array}$ \\
\hline 0.5 & 1.6393 & 0.5938 & 5.8450 & 0.0823 & 2.5237 & 0.7917 & 0.34425 & 1.0800 \\
\hline 0.7 & 2.2801 & 0.7840 & 7.9372 & 0.0432 & 4.0332 & 1.2832 & 0.55014 & 1.7504 \\
\hline 0.9 & 2.9126 & 1.2195 & 11.364 & 0.0255 & 6.4253 & 1.9614 & 0.87644 & 2.6755 \\
\hline 1.1 & 3.5370 & 1.3807 & 10.270 & 0.0193 & 8.3026 & 2.2643 & 1.1325 & 3.0886 \\
\hline 1.3 & 4.1534 & 1.5113 & 10.610 & 0.0155 & 10.2003 & 2.7026 & 1.3914 & 3.6865 \\
\hline 1.4 & 4.4586 & 1.6268 & 9.8367 & 0.0145 & 11.3604 & 2.7935 & 1.5496 & 3.8105 \\
\hline 1.6 & 5.0633 & 1.6801 & 11.812 & 0.0139 & 13.111 & 3.4764 & 1.7884 & 4.7419 \\
\hline
\end{tabular}

Fig. 5 gives straight lines from the relation between cathodic and anodic peak current $I_{p}$ against different concentrations of $\mathrm{Cu}^{2+}$ ions in $0.1 \mathrm{M} \mathrm{HCl}$. indicating diffusion process.

Two couples of copper ion peaks were obtained the first two cathodic peaks on the scan towards negative potentials and the second at reversal of scan shows two well defined anodic peaks (see Fig. 4). The cathodic couple of peaks are attributed to the reduction of $\mathrm{Cu}^{2+}$ to $\mathrm{Cu}^{+}$and then to $\mathrm{Cu}$ as cathodic mechanism. Then $\mathrm{Cu}$ transfers to $\mathrm{Cu}^{+}$and $\mathrm{Cu}^{+2}$ as anodic mechanism. Fig. 4 gave the effect of scan rate on the peak potential and peak current.

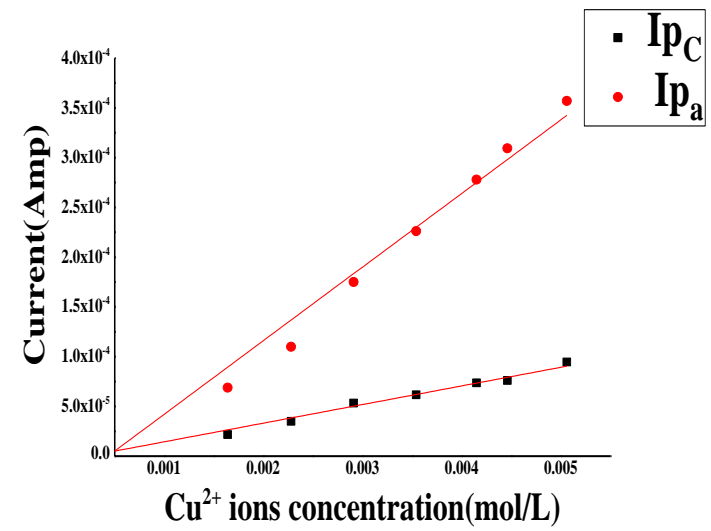

Fig.5: The relation between peak current Ip (Ipa - Ipc) against different $\mathrm{Cu}^{2+}$ ions concentration at $290.65 \mathrm{~K}$ and scan rate $0.1 \mathrm{~V} / \mathrm{S}$. 


\subsection{Effect of different scan rates}

Effect of different scan rates for the redox behavior of $\mathrm{Cu}^{2+}$ ions in $0.1 \mathrm{M} \mathrm{HCl}$ was studied at $(0.1,0.02,0.01)$ (V.s ${ }^{-1}$ ) and $290.65 \mathrm{~K}$ (Fig. 6). The solvation and kinetic parameters (Ep, Ip, $\Delta \mathrm{E}_{\mathrm{P}}, \mathrm{E}_{\mathrm{pc} / 2}, \mathrm{D}, \mathrm{k}_{\mathrm{S}}, \Gamma$ and $\mathrm{Q}$ ) of different scan rates of $\mathrm{Cu}^{2+}$ ions were presented in Table II (A, B).
The cathodic mechanism showed nearly constant peak current from the relation with square root of scan rate indicating it is diffusion controlled process while the anodic mechanism of dissolution of the deposited copper at the cathodic potential is a stripping process.

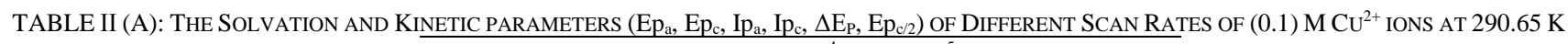

\begin{tabular}{ccccccc}
\hline$v$ & $\begin{array}{c}\mathrm{Ep}_{\mathrm{a}} \\
(\mathrm{v})\end{array}$ & $\begin{array}{c}\mathrm{Ep}_{\mathrm{c}} \\
(\mathrm{v})\end{array}$ & $\begin{array}{c}(-) \mathrm{Ip}_{\mathrm{a}} \times 10^{-4} \\
(\mathrm{~A})\end{array}$ & $\begin{array}{c}\mathrm{Ip}_{\mathrm{c}} \times 10^{-5} \\
(\mathrm{~A})\end{array}$ & $\begin{array}{c}\Delta \mathrm{E}_{\mathrm{P}} \\
(\mathrm{v})\end{array}$ & $\begin{array}{c}\mathrm{Ep}_{\mathrm{c} / 2} \\
(\mathrm{v})\end{array}$ \\
\hline 0.1 & 0.0553 & 0.2084 & 3.570 & 9.467 & 0.1532 & 0.1841 \\
0.02 & 0.0393 & 0.1785 & 2.219 & 3.760 & 0.1391 & 0.1563 \\
0.01 & 0.0352 & 0.160 & 1.795 & 2.858 & 0.1247 & 0.147 \\
\hline
\end{tabular}

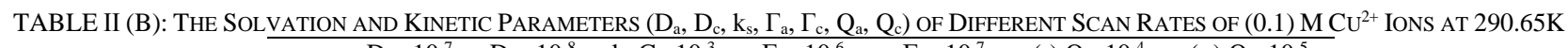

\begin{tabular}{cccccccc}
\hline$v$ & $\begin{array}{c}\mathrm{D}_{\mathrm{a}} \times 10^{-7} \\
\left(\mathrm{~cm}^{2} \cdot \mathrm{s}^{-1}\right)\end{array}$ & $\begin{array}{c}\mathrm{D}_{\mathrm{c} \times 10^{-8}} \\
\left(\mathrm{~cm}^{2} \cdot \mathrm{s}^{-1}\right)\end{array}$ & $\begin{array}{c}\mathrm{k}_{\mathrm{s}} \mathrm{C} \times 10^{-3} \\
\left(\mathrm{~cm}^{-1}\right)\end{array}$ & $\begin{array}{c}\Gamma_{\mathrm{a}} \times 10^{-6} \\
\left(\mathrm{~mol} / \mathrm{cm}^{2}\right)\end{array}$ & $\begin{array}{c}\Gamma_{\mathrm{c}} \times 10^{-7} \\
\left(\mathrm{~mol} / \mathrm{cm}^{2}\right)\end{array}$ & $\begin{array}{c}(-) \mathrm{Q}_{\mathrm{a}} \times 10^{-4} \\
(\mathrm{C})\end{array}$ & $\begin{array}{c}(+) \mathrm{Q}_{\mathrm{c}} \times 10^{-5} \\
(\mathrm{C})\end{array}$ \\
\hline 0.1 & 1.680 & 1.181 & 0.1393 & 1.311 & 3.476 & 1.788 & 4.742 \\
0.02 & 3.244 & 0.9319 & 4.379 & 4.074 & 6.905 & 5.557 & 9.418 \\
0.01 & 4.245 & 1.077 & 3.267 & 6.590 & 10.49 & 8.989 & 14.32 \\
\hline
\end{tabular}

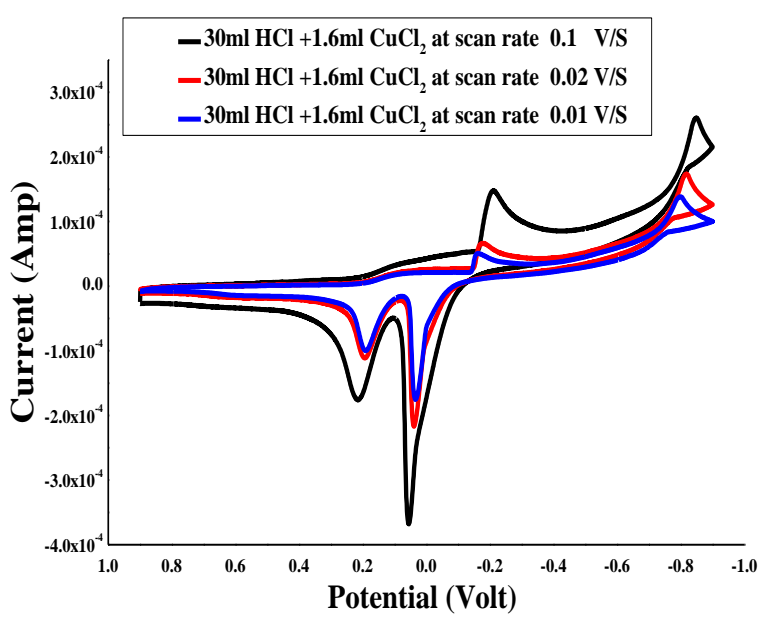

Fig. 6. Cyclic voltammogram of different scan rates of $\left(5.06 \times 10^{-3}\right) \mathrm{M} \mathrm{Cu}^{2+}$ ions in $0.1 \mathrm{M} \mathrm{HCL}$ at $290.65 \mathrm{~K}$.

2. Solvation and kinetic parameters in presence of Succinic hydrazide

2.1. Effect of different copper ions concentrations in presence of Succinic hydrazide

The electrochemical redox behavior of $\mathrm{Cu}^{2+}$ ions in the presence of Succinic hydrazide at the solid glassy carbon electrode was studied at the steady state current and cyclic waves were explained using equations (1-6) (Fig. 8). The solvation and kinetic parameters (Ep, Ip, $\Delta \mathrm{E}_{\mathrm{P}}, \mathrm{E}_{\mathrm{pc} / 2}, \mathrm{D}, \mathrm{k}_{\mathrm{S}}, \Gamma$ and $\mathrm{Q})$ of interaction of $\left(5.06 \times 10^{-3}\right) \mathrm{M} \mathrm{Cu}^{2+}$ ions and different concentrations of $\mathrm{Cu}^{2+}$ ions at $290.65 \mathrm{~K}$ and scan rate $0.1 \mathrm{~V} / \mathrm{S}$ were presented in Table III $(\mathrm{A}, \mathrm{B})$.

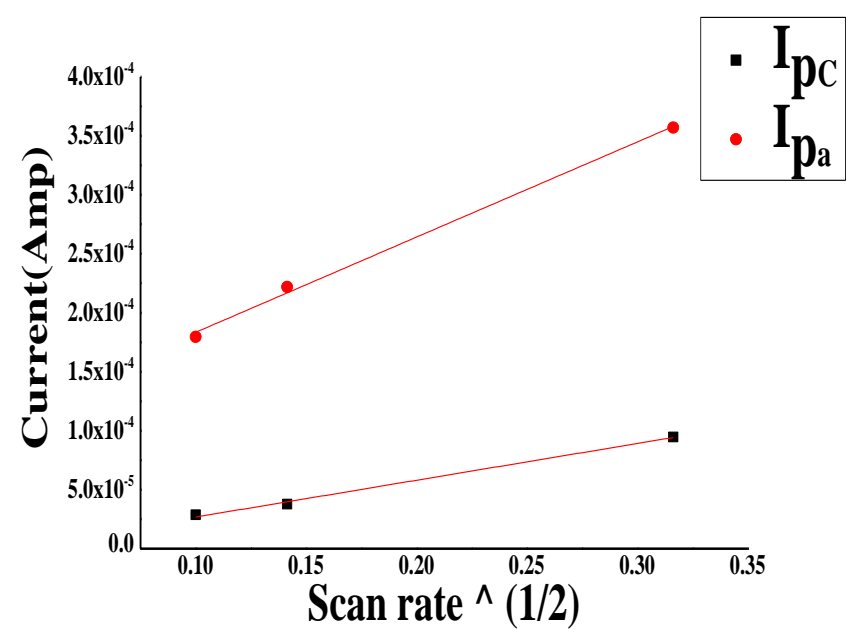

Fig. 7. The relation between peak current $I p\left(I_{p a}-I_{p c}\right)$ against the square root of different scan rates for $(0.1) \mathrm{M} \mathrm{Cu}^{2+}$ ions at $290.65 \mathrm{~K}$.

Randless Sevicek equation was used to apply the relation between cathodic and anodic peak current $I_{p}$ against different concentrations of $\mathrm{Cu}^{2+}$ ions in the presence of Succinic hydrazide (Fig. 9).

\subsection{Effect of different scan rates}

Effect of scan rate on the interaction between $\mathrm{Cu}^{2+}$ ions and Succinic hydrazide was studied in $(0.1,0.02$ and 0.01$)$ V.s. ${ }^{-1}$ (Fig. 10). The solvation and kinetic parameters (Ep, Ip, $\Delta \mathrm{E}_{\mathrm{P}}, \mathrm{Ep}_{\mathrm{c} / 2}, \mathrm{D}, \mathrm{k}_{\mathrm{S}}, \Gamma$ and $\left.\mathrm{Q}\right)$ of different scan rates of $\mathrm{Cu}^{2+}$ ions in the presence of Succinic hydrazide were presented in Table VI (A, B).

TABLE III (A): THe Solvation AND Kinetic PARAMETERs ( $\left.\mathrm{Ep}_{\mathrm{a}}, \mathrm{Ep}_{\mathrm{c}}, \mathrm{Ip}_{\mathrm{a}}, \mathrm{Ip}_{\mathrm{c}}, \Delta \mathrm{E}_{\mathrm{p}}, \mathrm{Ep}_{\mathrm{c} / 2}\right)$ OF INTERACTION OF $\left(5.06 \times 10^{-3}\right) \mathrm{M} \mathrm{CU}^{2+} \operatorname{IONS}_{\text {AND DiFFERENT }}$

\begin{tabular}{ccccccccccc}
\multicolumn{8}{c}{ CONCENTRATIONS OF SUCCINIC HYDRAZIDE AT 290.65 K AND SCAN RATE 0.1 V/S } \\
\hline $\mathrm{ml} \mathrm{0f} \mathrm{M}$ & $\begin{array}{c}{[\mathrm{M}] \times 10^{-3}} \\
\left(\mathrm{~mol} . \mathrm{L}^{-1}\right)\end{array}$ & Ml of L & $\begin{array}{c}{[\mathrm{L}] \times 10^{-5}} \\
\left(\mathrm{~mol}_{\mathrm{L}}^{-1}\right)\end{array}$ & $\begin{array}{c}\mathrm{Ep}_{\mathrm{a}} \\
(\mathrm{V})\end{array}$ & $\begin{array}{c}\mathrm{Ep}_{\mathrm{c}} \\
(\mathrm{V})\end{array}$ & $\begin{array}{c}(-) \mathrm{Ip}_{\mathrm{a}} \times 10^{-4} \\
(\mathrm{~A})\end{array}$ & $\begin{array}{c}\mathrm{Ip}_{\mathrm{c}} \times 10^{-5} \\
(\mathrm{~A})\end{array}$ & $\begin{array}{c}\Delta \mathrm{E}_{\mathrm{p}} \\
(\mathrm{V})\end{array}$ & $\begin{array}{c}\mathrm{Ep}_{\mathrm{c} / 2} \\
(\mathrm{~V})\end{array}$ \\
\hline 1.6 & 4.984 & 0.5 & 0.6307 & 0.0623 & 0.2061 & 3.419 & 10.99 & 0.1438 & 0.1798 \\
1.6 & 4.954 & 0.7 & 0.8775 & 0.0625 & 0.2063 & 3.175 & 10.39 & 0.1438 & 0.1806 \\
1.6 & 4.923 & 0.9 & 1.121 & 0.064 & 0.2029 & 2.950 & 9.958 & 0.139 & 0.1799 \\
1.6 & 4.893 & 1.1 & 1.362 & 0.0665 & 0.2058 & 2.786 & 9.403 & 0.1392 & 0.1818 \\
1.6 & 4.863 & 1.3 & 1.600 & 0.0665 & 0.2057 & 2.644 & 7.176 & 0.1392 & 0.1852 \\
1.6 & 4.848 & 1.4 & 1.718 & 0.0639 & 0.2093 & 2.606 & 7.554 & 0.1454 & 0.1872 \\
1.6 & 4.819 & 1.6 & 1.951 & 0.0614 & 0.2061 & 2.347 & 7.452 & 0.1448 & 0.1901 \\
1.6 & 4.420 & 4.6 & 5.145 & 0.0611 & 0.2186 & 2.156 & 4.449 & 0.1575 & 0.1987 \\
1.6 & 4.082 & 7.6 & 7.850 & 0.0528 & 0.2281 & 1.818 & 4.173 & 0.1753 & 0.2129 \\
\hline
\end{tabular}


TABLE III (B): THe SOlvation AND Kinetic PARAMETERS $\left(\mathrm{D}_{\mathrm{a}}, \mathrm{D}_{\mathrm{c}}, \mathrm{k}_{\mathrm{s}}, \Gamma_{\mathrm{a}}, \Gamma_{\mathrm{c}}, \mathrm{Q}_{\mathrm{a}}, \mathrm{Q}_{\mathrm{c}}\right.$ ) OF INTERACTION OF $\left(5.06 \times 10^{-3}\right) \mathrm{M} \mathrm{CU}^{2+}$ IONS AND DiFFERENT

\begin{tabular}{ccccccccc}
\multicolumn{7}{c}{ CONCENTRATIONS OF SUCCINIC HYDRAZIDE AT 290.65 K AND SCAN RATE 0.1 V/S } \\
\hline $\begin{array}{c}{[\mathrm{M}] \times 10^{-3}} \\
\left(\mathrm{~mol} . \mathrm{L}^{-1}\right)\end{array}$ & $\begin{array}{c}{[\mathrm{L}] \times 10^{-5}} \\
\left(\mathrm{~mol} . \mathrm{L}^{-1}\right)\end{array}$ & $\begin{array}{c}\mathrm{D}_{\mathrm{a}} \times 10^{-8} \\
\left(\mathrm{~cm}^{2} . \mathrm{s}^{-1}\right)\end{array}$ & $\begin{array}{c}\mathrm{D}_{\mathrm{c}} \times 10^{-9} \\
\left(\mathrm{~cm}^{2} . \mathrm{s}^{-1}\right)\end{array}$ & $\begin{array}{c}\mathrm{k}_{\mathrm{s}} \mathrm{C} \\
\left(\mathrm{cm}^{-1}\right)\end{array}$ & $\begin{array}{c}\Gamma_{\mathrm{a}} \times 10^{-7} \\
\left(\mathrm{~mol}^{-7} \mathrm{~cm}^{2}\right)\end{array}$ & $\begin{array}{c}\Gamma_{\mathrm{c}} \times 10^{-7} \\
\left(\mathrm{~mol}^{-7} \mathrm{~cm}^{2}\right)\end{array}$ & $\begin{array}{c}(-) \mathrm{Q}_{\mathrm{a}} \\
\times 10^{-4}(\mathrm{C})\end{array}$ & $\begin{array}{c}(+) \mathrm{Q}_{\mathrm{c}} \\
\times 10^{-5}(\mathrm{C})\end{array}$ \\
\hline 4.984 & 0.6307 & 15.90 & 16.42 & 0.0131 & 12.55 & 4.035 & 1.712 & 5.504 \\
4.954 & 0.8775 & 13.88 & 14.85 & 0.0126 & 11.66 & 3.814 & 1.590 & 5.202 \\
4.923 & 1.121 & 12.14 & 13.82 & 0.0117 & 10.83 & 3.657 & 1.478 & 4.988 \\
4.893 & 1.362 & 10.95 & 12.48 & 0.0109 & 10.23 & 3.453 & 1.395 & 4.710 \\
4.863 & 1.600 & 9.987 & 7.358 & 0.0091 & 9.709 & 2.635 & 1.324 & 3.595 \\
4.848 & 1.718 & 9.763 & 8.202 & 0.0104 & 9.570 & 2.774 & 1.305 & 3.784 \\
4.819 & 1.951 & 8.016 & 8.079 & 0.012 & 8.620 & 2.736 & 1.176 & 3.733 \\
4.420 & 5.145 & 8.039 & 3.423 & 0.009 & 7.917 & 1.634 & 1.080 & 2.228 \\
4.082 & 7.850 & 6.706 & 3.532 & 0.015 & 6.677 & 1.532 & 0.9108 & 2.090 \\
\hline
\end{tabular}

TABLE IV (A): THE SOLVATION AND KINETIC PARAMETERS (Ep $\mathrm{p}_{\mathrm{a}}, \mathrm{Ep}_{\mathrm{c}}, \mathrm{Ip}_{\mathrm{a}}, \mathrm{Ip}_{\mathrm{c}}, \Delta \mathrm{E}_{\mathrm{p}}, \mathrm{Ep}_{\mathrm{c} / 2}$ ) OF DIFFERENT SCAN RATES OF COPPER COMPLEX AT $290.65 \mathrm{~K}$

\begin{tabular}{ccccccccc}
\hline$v$ & $\begin{array}{c}{[\mathrm{M}] \times 10^{-3}} \\
\left(\mathrm{mol.} \mathrm{L}^{-1}\right)\end{array}$ & $\begin{array}{c}{[\mathrm{L}] \times 10^{-5}} \\
\left(\mathrm{mol.} \mathrm{L}^{-1}\right)\end{array}$ & \multicolumn{1}{c}{$\begin{array}{c}\mathrm{Ep}_{\mathrm{a}} \\
(\mathrm{v})\end{array}$} & \multicolumn{1}{c}{$\begin{array}{c}\mathrm{Ep}_{\mathrm{c}} \\
(\mathrm{v})\end{array}$} & $(-) \mathrm{I}_{\mathrm{pa}}(\mathrm{A}) \times 10^{-4}$ & $\mathrm{I}_{\mathrm{pc}}(\mathrm{A}) \times 10^{-5}$ & $\begin{array}{c}\Delta \mathrm{E}_{\mathrm{p}} \\
(\mathrm{v})\end{array}$ & $\begin{array}{c}\mathrm{Ep}_{\mathrm{c} / 2} \\
(\mathrm{v})\end{array}$ \\
\hline 0.1 & 4.082 & 7.850 & 0.0528 & 0.2281 & 1.8183 & 7.154 & 0.1404 & 0.2129 \\
0.02 & 4.082 & 7.850 & 0.048 & 0.2042 & 1.0199 & 3.104 & 0.1261 & 0.1924 \\
0.01 & 4.082 & 7.850 & 0.0455 & 0.1942 & 0.8749 & 2.442 & 0.1198 & 0.1775 \\
\hline
\end{tabular}

TABLE IV (B): THE SOLVATION AND KINETIC PARAMETERS $\left(\mathrm{D}_{\mathrm{a}}, \mathrm{D}_{\mathrm{c}}, \mathrm{k}_{\mathrm{s}}, \Gamma_{\mathrm{a}}, \Gamma_{\mathrm{c}}, \mathrm{Q}_{\mathrm{a}}, \mathrm{Q}_{\mathrm{c}}\right.$ ) OF DIFFERENT SCAN RATES OF COPPER COMPLEX AT $290.65 \mathrm{~K}$

\begin{tabular}{|c|c|c|c|c|c|c|c|c|c|}
\hline 0 & $\begin{array}{l}{[\mathrm{M}] \times 10^{-3}} \\
\left(\mathrm{~mol} . \mathrm{L}^{-1}\right)\end{array}$ & $\begin{array}{l}{[\mathrm{L}] \times 10^{-5}} \\
\left(\mathrm{~mol} . \mathrm{L}^{-1}\right)\end{array}$ & $\begin{array}{l}\text { Da x10-7 } \\
\left(\mathrm{cm}^{2} \cdot \mathrm{s}^{-1}\right)\end{array}$ & $\begin{array}{l}\text { De x10-8 } \\
\left(\mathrm{cm}^{2} \cdot \mathrm{s}^{-1}\right)\end{array}$ & $\begin{array}{c}\mathrm{k}_{\mathrm{s}} \mathrm{C} \times 10^{-3} \\
\left(\mathrm{~cm} \cdot \mathrm{s}^{-1}\right)\end{array}$ & $\begin{array}{c}\Gamma \mathrm{a} \times 10^{-6} \\
\left(\mathrm{~mol} / \mathrm{cm}^{2}\right)\end{array}$ & $\begin{array}{c}\Gamma \mathrm{c} \times 10^{-7} \\
\left(\mathrm{~mol} / \mathrm{cm}^{2}\right)\end{array}$ & $\begin{array}{r}(-) \mathrm{Qa} \\
\mathrm{x} 10^{-4}(\mathrm{C}) \\
\end{array}$ & $\begin{array}{c}(+) \mathrm{Qc} \\
\mathrm{x} 10^{-5}(\mathrm{C}) \\
\end{array}$ \\
\hline 0.1 & 4.082 & 7.850 & 0.6706 & 1.038 & 0.2574 & 0.677 & 2.627 & 0.9108 & 3.583 \\
\hline 0.02 & 4.082 & 7.850 & 1.055 & 0.9772 & 8.651 & 1.873 & 5.700 & 2.554 & 7.774 \\
\hline 0.01 & 4.082 & 7.850 & 1.553 & 1.209 & 4.916 & 3.213 & 8.966 & 4.382 & 12.23 \\
\hline
\end{tabular}

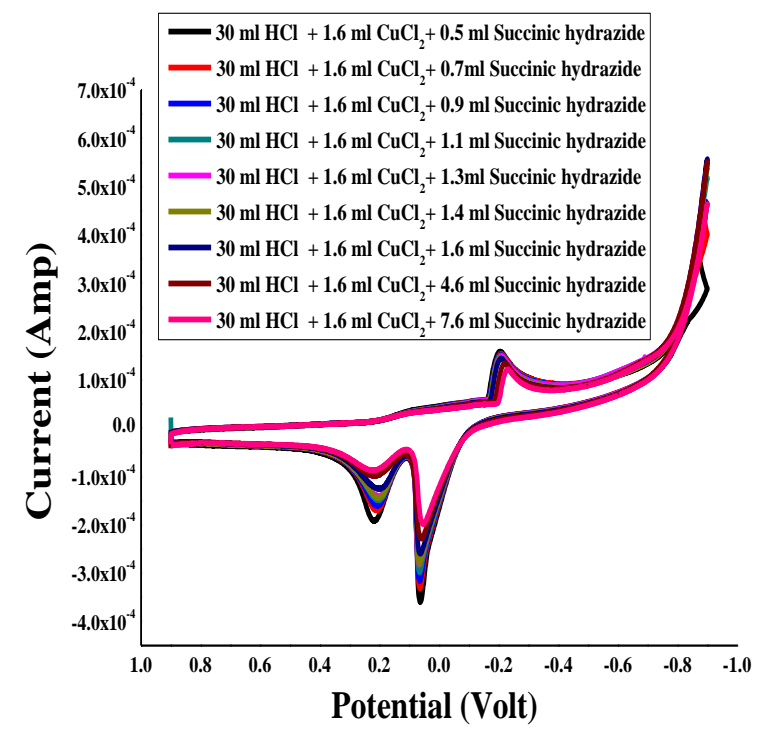

Fig. 8. Cyclic voltammograms for the interaction of $\left(5.06 \times 10^{-3}\right) \mathrm{M} \mathrm{Cu}^{2+}$ ions with different concentrations of Succinic hydrazide at $290.65 \mathrm{~K}$ and scan rate $0.1 \mathrm{~V} / \mathrm{S}$.

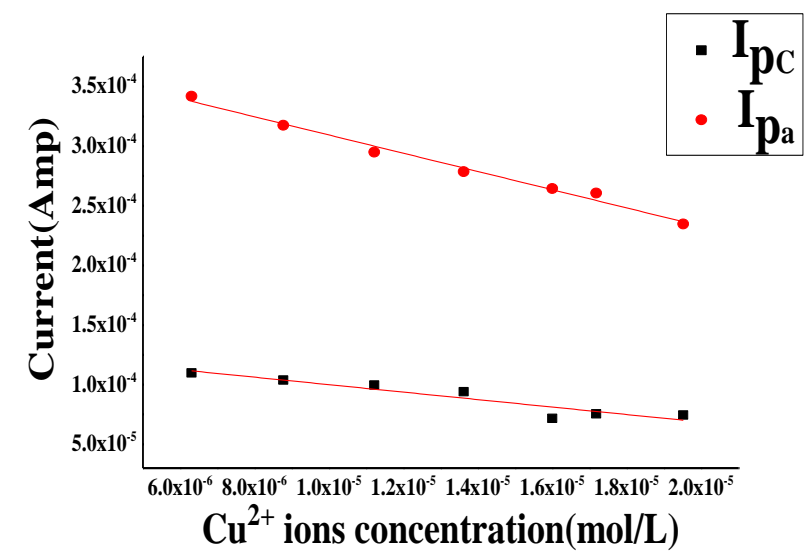

Fig. 9. The relation between peak current $\mathrm{Ip}\left(\mathrm{Ip}_{\mathrm{a}}-\mathrm{Ip}_{\mathrm{c}}\right)$ against different $\mathrm{Cu}^{2+}$ ions concentration in the presence of Succinic hydrazide at $290.65 \mathrm{~K}$ and scan rate $0.1 \mathrm{~V} / \mathrm{S}$.

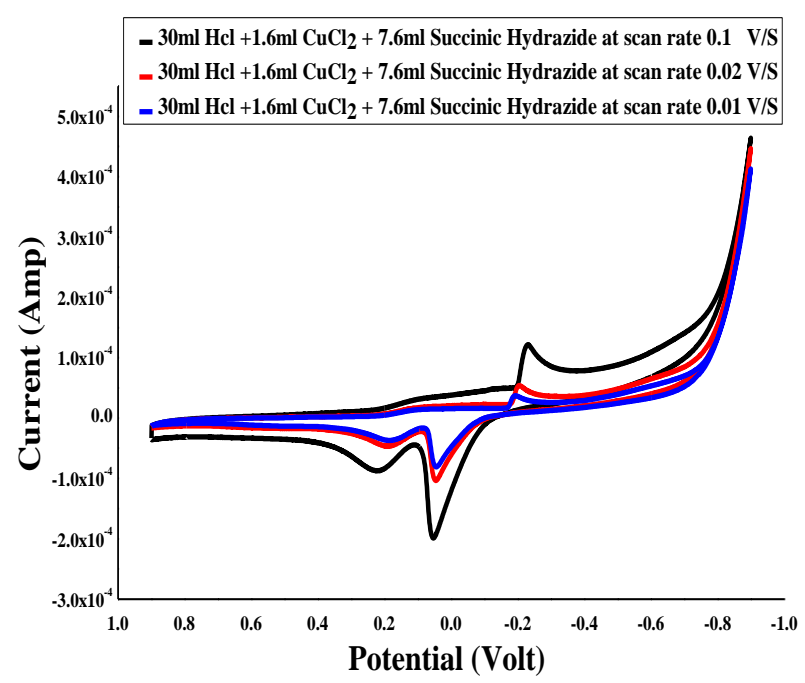

Fig. 10. Cyclic voltammogram of different scan rate of $\left(4.08 \times 10^{-3}\right) \mathrm{M} \mathrm{Cu}^{2+}$ ions with $\left(7.85 \times 10^{-5}\right) \mathrm{M}$ Succinic hydrazide at $290.65 \mathrm{~K}$.

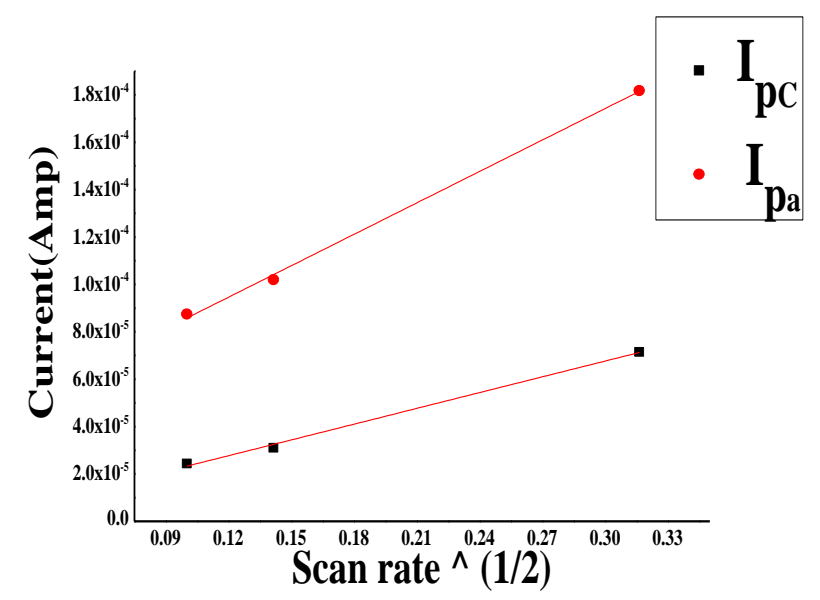

Fig. 11. The relation between peak current $I p\left(\mathrm{Ip}_{\mathrm{a}}-\mathrm{Ip}_{\mathrm{c}}\right)$ against different scan rates of copper complex at $290.65 \mathrm{~K}$. 


\section{Electrochemical behavior of the complexation between} $\mathrm{Cu} 2+$ ions and Succinic hydrazide

A stability constant is a measure of the strength of the interaction between the reagents that come together to form the complex.

The stability constants $\left(\beta_{\mathrm{j}}\right)$ for copper complex for each addition are calculated by applying the following equations [19-28].

$$
\begin{aligned}
& \Delta \mathrm{E}^{\circ}=\mathrm{E}^{\circ}{ }_{\mathrm{C}}-\mathrm{E}^{\circ}{ }_{\mathrm{M}}=2.303(\mathrm{RT} / \mathrm{nF}) *\left(\log \beta_{\mathrm{j}}+\mathrm{j} \log \mathrm{C}_{\mathrm{x}}\right) \\
& \mathrm{E}^{\circ}=\left(\mathrm{E}_{\mathrm{pa}}+\mathrm{E}_{\mathrm{pc}}\right) / 2
\end{aligned}
$$

$\Delta \mathrm{G}=-2.303 \mathrm{RT} \log \beta_{\mathrm{MX}}$

where, $\mathrm{E}_{\mathrm{M}}^{\circ}$ is the formal peak potential of metal at finally adding in the absence of Succinic hydrazide, $\mathrm{E}_{\mathrm{C}}^{\circ}$ is the formal peak potential of metal complex after each addition of Succinic hydrazide, $\mathrm{R}$ is a gas constant $\left(8.314 \mathrm{~J}_{\mathrm{mol}}{ }^{-1}\right.$. degree $\left.^{-1}\right), \mathrm{T}$ is the absolute temperature, $\mathrm{j}$ is the coordination number of the Stoichiometric complex, $C_{x}$ is the concentration of Succinic hydrazide in the solution, $\mathrm{E}^{\circ}$ is the formal potential, $\mathrm{E}_{\mathrm{pa}}$ is the anodic peak potential, $\mathrm{E}_{\mathrm{pc}}$ is the cathodic peak potential, $\beta_{\mathrm{j}}$ is the stability constant and $\Delta \mathrm{G}$ is the Gibbs free energy of interaction between $\mathrm{Cu}^{2+}$ ions and Succinic hydrazide.

The calculated values of $E^{\circ}, \beta_{j}$ and $\Delta G$ for copper complex are estimated and collected in Table $\mathrm{V}$.

The values of the stability constant and Gibbs free energy of complexation indicting that the interaction between copper ions and Succinic hydrazide is in the range of electrostatic interaction under the studying of concentration conditions.

TABle V: The Values of (Formal Potential Eº Stability Constant $\beta_{\mathrm{j}}$ And Gibbs Free Energy $\Delta$ G) for Copper Complex at 290.65 K and

\begin{tabular}{ccccccccc}
\multicolumn{10}{c}{ SCAN RATE 0.1 V/S } \\
\hline $\begin{array}{c}{[\mathrm{M}] \times 10^{-3}} \\
\left(\mathrm{~mol} . \mathrm{L}^{-1}\right)\end{array}$ & $\begin{array}{c}{[\mathrm{L}] \times 10^{-5}} \\
\left(\mathrm{~mol} . \mathrm{L}^{-1}\right)\end{array}$ & $\begin{array}{c}\mathrm{Ep}_{\mathrm{a}} \\
(\mathrm{V})\end{array}$ & $\begin{array}{c}\mathrm{Ep}_{\mathrm{c}} \\
(\mathrm{V})\end{array}$ & $\mathrm{j}$ & $\log [\mathrm{L}]$ & $\log \beta \mathrm{j}$ & $\beta \mathrm{j}$ & $\Delta \mathrm{G}(\mathrm{KJ} / \mathrm{mol})$ \\
\hline 4.984 & 0.6307 & 0.0623 & 0.2061 & 0.0013 & -5.2002 & 0.0193 & 1.045 & -0.1072 \\
4.954 & 0.8775 & 0.0625 & 0.2063 & 0.0018 & -5.0567 & 0.1668 & 1.468 & -0.9282 \\
4.923 & 1.121 & 0.064 & 0.2029 & 0.0023 & -4.9503 & 0.2464 & 1.763 & -1.3711 \\
4.893 & 1.362 & 0.0665 & 0.2058 & 0.0028 & -4.8658 & 0.2374 & 1.728 & -1.3213 \\
4.863 & 1.600 & 0.0665 & 0.2057 & 0.0033 & -4.7959 & 0.3807 & 2.403 & -2.1188 \\
4.848 & 1.718 & 0.0639 & 0.2093 & 0.0035 & -4.765 & 0.2057 & 1.606 & -1.1447 \\
4.819 & 1.951 & 0.0614 & 0.2061 & 0.004 & -4.7097 & 0.3236 & 2.107 & -1.8011 \\
4.420 & 5.145 & 0.0611 & 0.2186 & 0.0116 & -4.2886 & -0.08 & 0.832 & 0.4456 \\
4.082 & 7.850 & 0.0528 & 0.2281 & 0.0192 & -4.1051 & -0.331 & 0.467 & 1.8417 \\
\hline
\end{tabular}

\section{Biological activity (Antimicrobial and Antifungal) activity}

The antimicrobial activity was estimated based on inhibition zone size formed around discs of Succinic hydrazide and its complex on a petri dish with Luria Bertani agar (LB-agar) plates to measure the efficacy of the used compounds [29-34].

\subsection{Antibacterial activity}

The antibacterial activity of copper complex was compared with the activity of Succinic hydrazide using Enterobacter and Staphylococcus aureus (gram-negative bacteria) (Table VI). Presence of higher inhibition zone was observed around disc of copper complex with $(9,11) \mathrm{mm}$, respectively while absence of it around disc of Succinic hydrazide. So, it can act as bacteriostatic agent towards these bacteria (Fig. 13).

Table VI: Inhibition zone disc $(\mathrm{mm})$ of Succinic hydrazide and copper complex with gram-negative bacteria and Fungi.

\begin{tabular}{cccc}
\hline \multirow{2}{*}{ Solid } & \multicolumn{3}{c}{ Inhibition zone disc (mm) } \\
\cline { 2 - 4 } & Types of gram-negative bacteria & $\begin{array}{c}\text { Type of } \\
\text { Fungi }\end{array}$ \\
\cline { 2 - 4 } & Enterobacter & $\begin{array}{c}\text { Staphylococcus } \\
\text { aureus }\end{array}$ & $\begin{array}{c}\text { Candida } \\
\text { albicans }\end{array}$ \\
\hline Succinic hydrazide & Absence & Absence & $\begin{array}{c}\text { Absence } \\
\text { Copper complex }\end{array}$ \\
\hline
\end{tabular}

\subsection{Antifungal activity}

Also, the experimental antifungal activity of copper complex was compared with the activity of Succinic hydrazide using Candida albicans (Table VI) indicating formation of higher inhibition zone $(7 \mathrm{~mm})$ around disc of copper complex only so, it can be used as antifungal drug (Fig. 14).

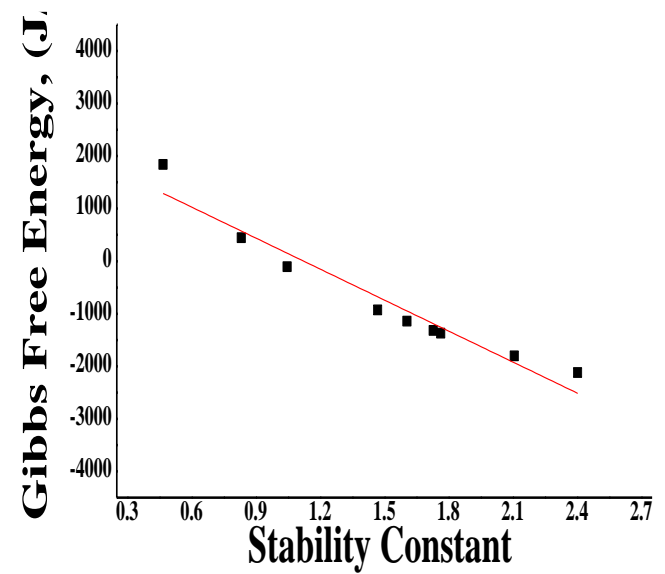

Fig. 12. The relation between Gibbs free energy $(\Delta \mathrm{G})$ against stability constant $\left(\beta_{\mathrm{MX}}\right)$ at $290.65 \mathrm{~K}$ and scan rate $0.1 \mathrm{~V} / \mathrm{S}$.
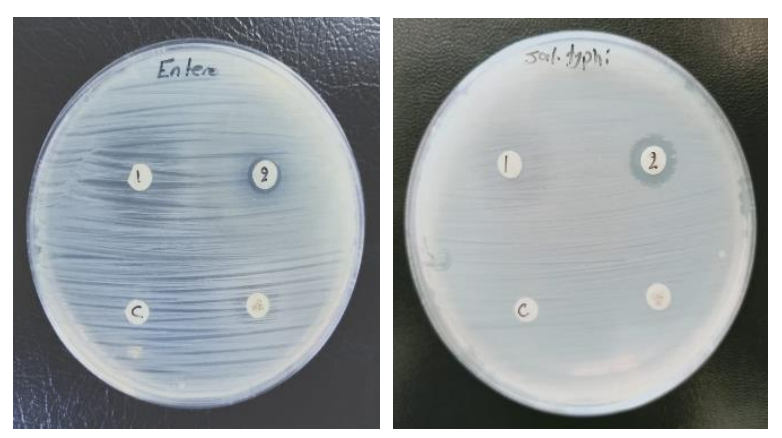

Fig. 13. Inhibition zone discs (6mm) of [(1) Succinic hydrazide (2) copper complex] on the surface of an LB-agar plate with (a) Enterobacter (b) Staphylococcus aureus. 


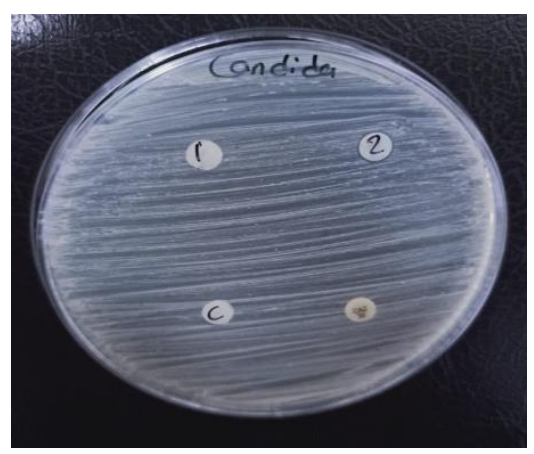

Fig. 14. Inhibition zone discs (6mm) of [(1) Succinic hydrazide (2) copper complex] on the surface of an LB-agar plate with Candida albicans.

\section{REFERENCES}

[1] G. A. Mabbott, An Introduction to Cyclic Voltammetry, J. Chem. Educ.,60, 1983, 697-702.

[2] J. M. Bobbitt, and Wills, P. John, J.Org.Chem.,45,1978 (1980).

[3] Nelsen, F. Stephen, Kessel, R. Carl, Brien, J. David, and Weinhold. Frank, J. Org.Chem.,45, 2116(1980).

[4] Powers, J. Michael, and Meyer, J. Thomas, J. Amer Chem. Soc, 102, 1289 (1980).

[5] K. Kalyanasundaram, J. Kiwi, M. Gratze1, Helv. Chim. Acta, 61, 2720 (1978).

[6] C. A. Rice, and J. T. Spence, Inorg. Chem, 19, 2845 (1980).

[7] L. Trakova, L. Zerzankova, F. Dyeka, Mikelova R. and F. Jelen, Study of copper and purine -copper complexes on modified electrodes by cyclic voltammetry, Sensors, 2008, 8, 429-444.

[8] C.H. Bamford, R.G. Compton, Electrode Kinetics: Principles and Methodology, Vol.26, 1st Edition, Elsevier Science, 1986.

[9] D.A.C. Brownson, C. E. Banks, The Handbook of Graphene Electrochemistry, Springer-Verlag London Ltd., 2014.

[10] D.K. Gosser, Cyclic Voltammetry: Simulation and analysis of reaction mechanisms, New York, VCH, 1993.

[11] P. Zanello, Inorganic Electrochemistry Theory, Practice and Application, The Royal Society of Chemistry, 2003.

[12] T. Kissinger Peter and R. Heineman William, Cyclic voltammetry, J. Chem. Edu, $1983,60,9,702-706$.

[13] H. Matsuda, \&. Y.Z. Ayabe; The theory of the cathode-ray polarography of Randles-Sevcik, Electrochem.; 1955; 59 (1955) 494-503.

[14] A. A. El-Khouly, E.A. Gomaa, and S. Abou El-leef, Bull Electrochem.;19(4), 2003,153-164.

[15] E.A. Gomaa, E.M. Abou Elleef, and M. H. Mahmoud, Eur Chem Bull., 2(10), 2013, 732-735.

[16] E.A. Gomaa, S. E. Salem, American Association for Science and Technology Communications),3(3), 2016; 160-168.

[17] M. M. El-Defrawy, E.A. Gomaa, S. E. Salem, F.M. Abdel Razek, Progress in Chemical and Biochemical Research, 1, 2018, 11-18.

[18] S.E. El-Shereafy, E.A. Gomaa, A.M. Yousif, A.S.A. El-Yazed; Electrochemical and thermodynamic estimations of the interaction parameters for bulk and nano-siilver nitrate (NSN) with cefdinir drug using glassy carbon electrode.; Iranian Journal of Materials Science \& Engineering, 2017;14(4) ;48-57.

[19] G.A. Mabbott; An introduction to cyclic voltammetry; Journal of Chemical Education, 1983, 60(9), 697-702.

[20] C.M.A. Brett, A.M.O. Brett, Electrochemistry Principles, methods and application, Oxford University Press, Oxford, 1993.

[21] C.H., C.F, H. Tipper, R, G. Compton; Electrode Kinetics: Principles and methodology (Vol.26)., 1986; Elsevier, New York.

[22] Brownson D.A.C., C.E. Banks; The Handbook of Graphene Electrochemistry.; eBook; 2014.

[23] D.K. Grosser, Cyclic voltammetry: simulation and analysis of reaction mechanisms (Vol.43). VCH; New York 1993.

[24] R. Shalini, A.K. Shukla; N. Munichandraiah; A cyclic voltammetric study of the kinetics and mechanism of electrodeposition of manganese dioxide, Journal of Applied Electrochemistry, 1998,28,1235-1241.

[25] M.N. El-Hady, E.A. Gomaa, A.G. Al-Harazie, Cyclic Voltammetry of bulk and nano $\mathrm{Cd}$ in presence of Ceftazidime and some DFT calculations; J Mol Liq., 2019;276:970-985.

[26] R.S. Nicholson, I. Shain; Theory and Application of Cyclic Voltammetry for Measurement of Electrode Reaction Kinetics.; Analytical Chemistry, 1965; 37(2), 178-190.
[27] E.A. Gomaa, M.H. Mahmoud, M.G. Mousa, E.M. El-Dahshan; Cyclic Voltammetry for the interaction between Bimthus Nitrate and Methyl Red in Potassium Nitrate Solutions; Chemical Methodologies, 2018, 3(1.pp. 1-144), 1-11.

[28] E.A. Gomaa, R.M. Abu-Qarn; Ionic association and thermodynamic parameters for solvation of vanadyl sulfate in ethanol-water mixtures at different temperatures; Journal of molecular liquids, 2017; 232, 319-324.

[29] T. Johnson and C. Case, 1995. "Chemical Methods of Control," adapted from Laboratory Experiments in Microbiology, Brief Edition, 4th ed. Redwood City, CA: Benjamin/Cummings Publishing Co.

[30] V. H. Baker and Tokuhisa, 2004. "What is a zone of inhibition on an agar plate?", Molecular Biology Archive, University of Chicago and Argonne National Laboratories, Newton Bulletin Board System [accessed September 11, 2006]

[31] D.M. Rollins and S.W. Joseph, 2000; "Antibiotic Disk Susceptibilities," Department of Cell Biology and Molecular Genetics, University of Maryland, College Park [accessed September 11, 2006].

[32] E. A. Gomaa, S. E. Salem, American Association for Science and Technology Communications),3(3), 2016; 132-138.

[33] J.S. Glasser, C.H. Guymon, K. Mende, S.E. Wolf, D.R. Hospenthal, C.K. Murray; Activity of topical antimicrobial agents against multidrug-resistant bacteria recovered from burn patients. Burns 2010: 36 (8): 1172-84.

[34] T.E. Taddonio, P.D. Thomson, D.J. Smith, J.K. Prasad; Topical antimicrobial testing of burn wound microorganisms: evaluation of a new technique; Burns 1990: 16 (1): 64-66. 